\title{
Exotic meson decays in the environment with chiral imbalance
}

\author{
A. A. Andrianov ${ }^{1,2, \star}$ V. A. Andrianov ${ }^{1, \star \star}$ D. Espriu ${ }^{2, \star \star \star}$ A.V. lakubovich ${ }^{1, \star \star \star \star}$ and A.E. Putilova ${ }^{1, \dagger}$ \\ ${ }^{1}$ Faculty of Physics, Saint Petersburg State University, Universitetskaya nab. 7/9, Saint Petersburg 199034, \\ Russia, and \\ ${ }^{2}$ Departament de Física Quàntica i Astrofísica and Institut de Ciéncies del Cosmos (ICCUB), Universitat de \\ Barcelona, Martí i Franqués 1, 08028 Barcelona, Spain
}

\begin{abstract}
An emergence of Local Parity Breaking (LPB) in central heavy-ion collisions (HIC) at high energies is discussed. LPB in the fireball can be produced by a difference between the number densities of right- and left-handed chiral fermions (Chiral Imbalance) which is implemented by a chiral (axial) chemical potential. The effective meson lagrangian induced by QCD is extended to the medium with Chiral Imbalance and the properties of light scalar and pseudoscalar mesons $\left(\pi, a_{0}\right)$ are analyzed. It is shown that exotic decays of scalar mesons arise as a result of mixing of $\pi$ and $a_{0}$ vacuum states in the presence of chiral imbalance. The pion electromagnetic formfactor obtains an unusual parity-odd supplement which generates a photon polarization asymmetry in pion polarizability. We hope that the above pointed indications of LPB can be identified in experiments on LHC, RHIC, CBM FAIR and NICA accelerators.
\end{abstract}

\section{Topological charge, Chiral Imbalance and axial chemical potential}

The behaviour of baryonic matter under extreme conditions has got recently a lot of interest [1,2]. A medium generated in the heavy ion collisions may serve for detailed studies, both experimental and theoretical, of various phases of hadron matter. In this context new properties of QCD in the hot and dense environment are tested in current accelerator experiments on RHIC and LHC $[3,4]$.

In heavy ion collisions, in principle, there are two distinct experimental situations for peripheral and central collisions. In the first case the so-called Chiral Magnetic Effect (CME) can be detected, details see in [5] and also [6] for a review and additional references.

In the second case there are some experimental indications of an abnormal dilepton excess in the range of low invariant masses and rapidities and moderate values of the transverse momenta [7]-[11] (see the reviews in [12]), which can be thought of as a result of LPB in the medium (the details can be found in [13]). In particular, in heavy-ion collisions at high energies, with raising temperatures and baryon densities, metastable states can appear in the finite-volume fireball with a nontrivial topological

\footnotetext{
${ }^{\star}$ e-mail: andrianov@icc.ub.edu

$\star \star$ e-mail: v.andriano@rambler.ru

$\star \star \star$ e-mail: espriu@icc.ub.edu

$\star \star \star \star$ e-mail: a.yakubovich@outlook.com

†e-mail: alyput@gmail.com
} 
axial charge (due to fluctuations of gluonic fields) $T_{5}$, which is related to the gluon gauge field $G_{i}$,

$$
T_{5}(t)=\frac{1}{8 \pi^{2}} \int_{\text {vol. }} d^{3} x \varepsilon_{j k l} \operatorname{Tr}\left(G^{j} \partial^{k} G^{l}-i \frac{2}{3} G^{j} G^{k} G^{l}\right), \quad j, k, l=1,2,3,
$$

where the integration is over the fireball volume. Its jump $\Delta T_{5}$ can be associated with the space-time integral of the gauge-invariant Chern-Pontryagin density,

$$
\begin{aligned}
\Delta T_{5} & =T_{5}\left(t_{f}\right)-T_{5}(0)=\frac{1}{16 \pi^{2}} \int_{0}^{t_{f}} d t \int_{\text {vol. }} d^{3} x \operatorname{Tr}\left(G^{\mu v} \widetilde{G}_{\mu v}\right)=\frac{1}{4 \pi^{2}} \int_{0}^{t_{f}} d t \int_{\text {vol. }} d^{3} x \partial^{\mu} K_{\mu}, \\
K_{\mu} & =\frac{1}{2} \epsilon_{\mu \nu \rho \sigma} \operatorname{Tr}\left(G^{v} \partial^{\rho} G^{\sigma}-i \frac{2}{3} G^{v} G^{\rho} G^{\sigma}\right) .
\end{aligned}
$$

For the time being we adopt a static case and neglect a topological current flux through the fireball boundary during lifetime of the corresponding thermodynamic phase in a domain.

It is known that the divergence of isosinglet axial quark current $J_{5, \mu}=\bar{q} \gamma_{\mu} \gamma_{5} q$ is locally constrained via the relation of partial conservation of axial current affected by the gluon anomaly,

$$
\partial^{\mu} J_{5, \mu}-2 \widehat{i m}_{q} J_{5}=\frac{N_{f}}{2 \pi^{2}} \partial^{\mu} K_{\mu} ; \quad J_{5}=\bar{q} \gamma_{5} q
$$

This relation allows to find the relation of a nonzero topological charge with a non-trivial quark axial charge $Q_{5}^{q}$. Namely, integrating over a finite volume of fireball we come to the equality,

$$
\begin{aligned}
& \frac{d}{d t}\left(Q_{5}^{q}-2 N_{f} T_{5}\right) \simeq 2 i \int_{\text {vol. }} d^{3} x \widehat{m}_{q} \bar{q} \gamma_{5} q, \\
& Q_{5}^{q}=\int_{\text {vol. }} d^{3} x q^{\dagger} \gamma_{5} q=\left\langle N_{L}-N_{R}\right\rangle,
\end{aligned}
$$

where $\left\langle N_{L}-N_{R}\right\rangle$ stands for the vacuum averaged difference between left and right chiral densities of baryon number. Therefrom it follows that in the chiral limit (when the masses of light quarks are taken zero) the axial quark charge is conserved in the presence of non-zero (metastable) topological charge. If for the lifetime of fireball and the size of hadron fireball of order $L=5-10 \mathrm{fm}$, the average topological charge is non-zero, $\left\langle\Delta T_{5}\right\rangle \neq 0$, then it may be associated with a topological chemical potential $\mu_{T}$ or an axial chemical potential $\mu_{5}$ [14] for neglected masses of light $u, d$ quarks. Thus we have,

$$
\left\langle\Delta T_{5}\right\rangle \simeq \frac{1}{2 N_{f}}\left\langle Q_{5}^{q}\right\rangle \Longleftrightarrow \mu_{5} \simeq \frac{1}{2 N_{f}} \mu_{T},
$$

Thus adding to the QCD lagrangian the term $\Delta \mathcal{L}_{\text {top }}=\mu_{T} \Delta T_{5}$ or $\Delta \mathcal{L}_{q}=\mu_{5} Q_{5}^{q}$, we get the possibility of accounting for non-trivial fluctuations of topological charge (fluctons) in the nuclear (quark) fireball.

In the general, Lorentz covariant form the field dual to the fluctons is described by means of the classical pseudoscalar field $a(x)$, so that,

$$
\Delta \mathcal{L}_{a}=\frac{N_{f}}{2 \pi^{2}} K_{v} \partial^{v} a(x) \simeq \frac{1}{\pi^{2}} K_{v} b^{v} \Longleftrightarrow b^{v} \bar{q} \gamma_{v} \gamma_{5} q, \quad b_{v} \simeq\left\langle\partial_{\nu} a(x)\right\rangle \simeq \text { const. }
$$

Thus in a quasi-equilibrium situation the appearance of a nearly conserved chiral charge can be incorporated with the help of an axial (chiral) vector chemical potential $b_{v}$. The appearance of a space vector part in $b_{v}$ can be associated with the non-equilibrium axial charge flow [15-17]. 


\section{QCD-inspired effective meson Lagrangian for $S U_{f}(2)$ case}

For the detection of Local Parity Breaking in the hadron fireball we implement the generalized sigma model with a background 4-vector of axial chemical potential [18], symmetric under $S U_{L}\left(N_{f}\right) \times$ $S U_{R}\left(N_{f}\right)$, for $u, d$-quarks $\left(N_{f}=2\right)$,

$$
\begin{aligned}
L & =\frac{1}{4} \operatorname{Tr}\left(D_{\mu} H\left(D^{\mu} H\right)^{\dagger}\right)+\frac{B}{2} \operatorname{Tr}\left[m\left(H+H^{\dagger}\right)\right]+\frac{M^{2}}{2} \operatorname{Tr}\left(H H^{\dagger}\right) \\
& -\frac{\lambda_{1}}{2} \operatorname{Tr}\left[\left(H H^{\dagger}\right)^{2}\right]-\frac{\lambda_{2}}{4}\left[\operatorname{Tr}\left(H H^{\dagger}\right)\right]^{2}+\frac{c}{2}\left(\operatorname{det} H+\operatorname{det} H^{\dagger}\right),
\end{aligned}
$$

where $H=\xi \Sigma \xi$ is an operator for meson fields, $m$ is an average mass of current quarks, $M$ is a "tachyonic" mass generating the spontaneous breaking of chiral symmetry, $B, c, \lambda_{1}, \lambda_{2}$ are real constants.

The matrix $\Sigma$ includes the singlet scalar meson $\sigma$, its vacuum average $v$ and the isotriplet of scalar mesons $a_{0}^{0}, a_{0}^{-}, a_{0}^{+}$,

$$
\Sigma=\left[\begin{array}{cc}
v+\sigma+a_{0}^{0} & \sqrt{2} a_{0}^{+} \\
\sqrt{2} a_{0}^{-} & v+\sigma-a_{0}^{0}
\end{array}\right]
$$

The operator $\xi$ realizes a nonlinear representation of the chiral group and is determined by the isotriplet $\pi^{0}, \pi^{-}, \pi^{+}$of pseudoscalar mesons,

$$
\begin{gathered}
\xi=\exp \left(\frac{i \vec{\pi} \vec{\tau}}{2 f_{\pi}}\right) \approx 1+\frac{i \vec{\pi} \vec{\tau}}{2 f_{\pi}}-\frac{(\vec{\pi} \vec{\tau})^{2}}{8 f_{\pi}^{2}}, \\
\vec{\pi} \vec{\tau}=\left[\begin{array}{cc}
\pi^{0} & \sqrt{2} \pi^{+} \\
\sqrt{2} \pi^{-} & -\pi^{0}
\end{array}\right],
\end{gathered}
$$

where $\vec{\tau}$ are Pauli matrix, $f_{\pi}$ is a decay constant of $\pi$ mesons.

The covariant derivative of $H$ contains external gauge fields $R_{\mu}$ and $L_{\mu}$,

$$
D_{\mu} H=\partial_{\mu} H-i L_{\mu} H+i H R_{\mu}
$$

These fields include the photon field $A_{\mu}$ and are supplemented also a background 4-vector of axial chemical potential $\left(b_{\mu}\right)=\left(b_{0}, \mathbf{b}\right)$,

$$
\begin{aligned}
& R_{\mu}=e Q_{e m} A_{\mu}-b_{\mu} \cdot 1_{2 \times 2}, \\
& L_{\mu}=e Q_{e m} A_{\mu}+b_{\mu} \cdot 1_{2 \times 2},
\end{aligned}
$$

where $Q_{e m}=\frac{1}{2} \tau_{3}+\frac{1}{6} 1_{2 \times 2}$ is a matrix of electromagnetic charge.

The complete effective meson lagrangian has to include a P-odd part the Wess-Zumino-Witten effective action [19] which is modified in the chirally imbalanced medium. The relevant parts of WZW action read,

$$
\Delta \mathcal{L}_{W Z W}=-\frac{i e N_{c} b_{v}}{6 \pi^{2} v^{2}} \epsilon^{\nu \sigma \lambda \rho} A_{\rho}\left(\partial_{\sigma} \pi^{+}\right)\left(\partial_{\lambda} \pi^{-}\right)-\frac{e^{2} N_{c}}{24 \pi^{2} v} \epsilon^{\nu \sigma \lambda \rho}\left(\partial_{\sigma} A_{\lambda}\right)\left(\partial_{v} A_{\rho}\right) \pi^{0}
$$




\section{Chiral (scalar) condensate depending on chiral chemical vector}

The mass gap equation for the scalar condensate follows from (7),

$$
-4\left(\lambda_{1}+\lambda_{2}\right) v^{3}+\left(2 M^{2}+4 b^{2}+2 c\right) v+2 B m=0 .
$$

The general solution of this equation reads

$$
\begin{aligned}
v\left(b_{\mu}\right) & =\frac{1}{6^{\frac{2}{3}}\left(\lambda_{1}+\lambda_{2}\right)}\left[9 B m\left(\lambda_{1}+\lambda_{2}\right)^{2}\right. \\
& \left.+\sqrt{3\left(\lambda_{1}+\lambda_{2}\right)^{3}\left(27 B^{2} m^{2}\left(\lambda_{1}+\lambda_{2}\right)-2\left(M^{2}+2 b^{2}+c\right)^{3}\right)}\right]^{1 / 3} \\
& +\frac{M^{2}+2 b^{2}+c}{6^{1}}\left[9 B m\left(\lambda_{1}+\lambda_{2}\right)^{2}\right. \\
& \left.+\sqrt{3\left(\lambda_{1}+\lambda_{2}\right)^{3}\left(27 B^{2} m^{2}\left(\lambda_{1}+\lambda_{2}\right)-2\left(M^{2}+2 b^{2}+c\right)^{3}\right)}\right]^{-1 / 3} .
\end{aligned}
$$

There are different regions for chiral vector covariant under Lorentz transformations of fireball frame.

1. Chiral charge imbalance region when $b^{2}>0$, in the rest frame the chiral background $\left(b^{\mu}\right)=$ $\left(\mu_{5}, 0,0,0\right)$.

2. Chiral vector imbalance region with $b^{2}<0$, in the static frame the chiral background is taken along the beam axis $\left(b^{\mu}\right)=(0,0,0, b)$.

3. Transition region with $b^{2}=0$, in the light-cone background $\left(b^{\mu}\right)=(b, 0,0, \pm b)$.

We stress that in a hot medium Lorentz invariance is broken by thermal bath and the physical effects depend on a particular set of components of $\left(b^{\mu}\right)$.

The plots for condensates display the enhancement of CSB and the restoration of chiral symmetry depending on the sign of $b_{\mu} b^{\mu}$.
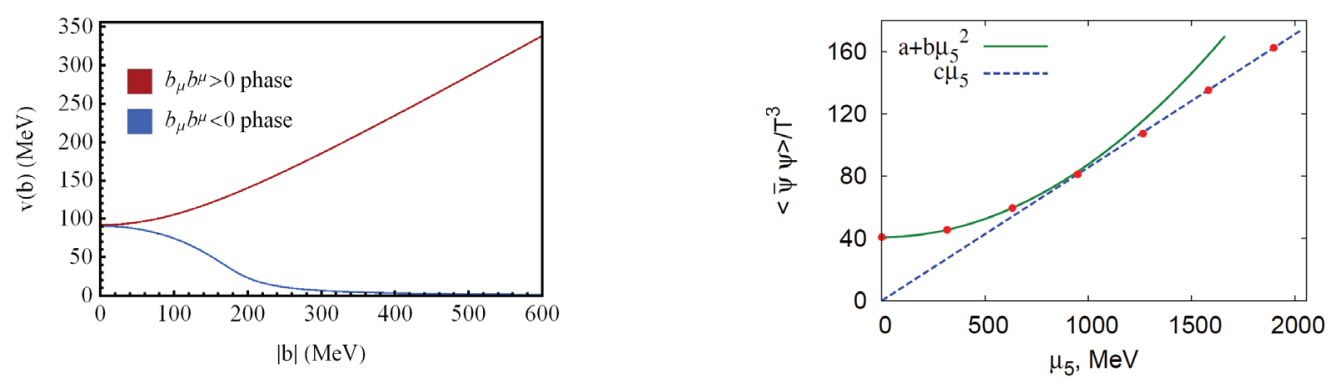

Figure 1. Left: the condensate in $b_{\mu} b^{\mu}>0$ and $b_{\mu} b^{\mu}<0$ region; right: the vacuum average $v\left(\mu_{5}\right) \sim\langle\bar{\psi} \psi\rangle / B$ from the lattice calculations [20]. 
Namely, in the chiral imbalance region with $b^{2}>0$ the increasing of chemical potentials causes the growth of chiral condensate, i.e. enhances Chiral Symmetry Breaking (CSB).

Instead, in the chiral vector imbalance region with $b^{2}<0$ the chiral condensate is decreasing with growing $\left|b^{2}\right|$ up to $\left|b^{2}\right|=\frac{1}{2}\left(M^{2}+c\right)$. At this scale in the chiral limit $m \rightarrow 0$ the CSB parameter $v \rightarrow 0$ (see below) and spontaneous CSB is restored.

At last the CSB parameter $v$ is insensitive to the light-cone $b^{\mu}$.

For $b^{2}>0$ in the rest frame of vector $\left(b_{\mu}\right)=\left(\mu_{5}, 0,0,0\right)$ one can compare the predictions of our effective meson lagrangian (see Fig.1, the left plot) with the lattice estimations [20] (see Fig.1, the right plot) which clearly shows the enhancement of CSB.

However non-zero real space components of $\left(b_{\mu}\right)$ produce non-Hermitian purely imaginary vertices after euclidization of QCD which makes it difficult to compute on the lattice their contribution to the quark determinant.

\section{Meson mass spectrum in different chiral imbalance regions}

Introduce the definitions for meson state masses in the chiral imbalance environment. The mass matrix for scalar and pseudoscalar mesons on the diagonal takes the following values,

$$
\begin{aligned}
& m_{a}^{2}=-2\left(M^{2}-2\left(3 \lambda_{1}+\lambda_{2}\right) v^{2}-c+2 b^{2}\right), \\
& m_{\sigma}^{2}=-2\left(M^{2}-6\left(\lambda_{1}+\lambda_{2}\right) v^{2}+c+2 b^{2}\right), \\
& m_{\pi}^{2}=\frac{2 B m}{v} .
\end{aligned}
$$

After diagonalization we define distorted masses as $m_{e f f+}$ for the field $\tilde{a}$ and $m_{e f f-}$ for the field $\tilde{\pi}$

$$
m_{e f f \pm}^{2}=\frac{1}{2}\left(m_{a}^{2}+m_{\pi}^{2} \pm \sqrt{\left(m_{a}^{2}-m_{\pi}^{2}\right)^{2}+\left(8 b^{\mu} k_{\mu}\right)^{2}}\right) .
$$

\subsection{Parameters of QCD-inspired generalized sigma model}

Let us normalize the vacuum parameters of our model. We take $m_{\pi}=139 \mathrm{MeV}, m_{a}=980 \mathrm{MeV}$, $m_{\sigma}=500 \mathrm{MeV}, m=5.5 \mathrm{MeV}, \mu_{5}=0, M=300 \mathrm{MeV}, v=92 \mathrm{MeV}$. Then from the following Eqs.

$$
\left\{\begin{aligned}
m_{\sigma}^{2} & =-2\left(M^{2}-6\left(\lambda_{1}+\lambda_{2}\right) v^{2}+c\right) \\
m_{a}^{2} & =-2\left(M^{2}-2\left(3 \lambda_{1}+\lambda_{2}\right) v^{2}-c\right) \\
m_{\pi}^{2} & =\frac{2 B m}{v} \\
v\left(\mu_{5}=0\right) & =\sqrt{\frac{M^{2}+c}{2\left(\lambda_{1}+\lambda_{2}\right)}}+\frac{B m}{2\left(M^{2}+c\right)}
\end{aligned}\right.
$$

one can find $\lambda_{1}, \lambda_{2}, c$ and $b$. We have for parameters $\lambda_{1}=1.64850 \times 10, \lambda_{2}=-1.31313 \times 10$, $c=-4.46874 \times 10^{4} \mathrm{MeV}^{2}, B=1.61594 \times 10^{5} \mathrm{MeV}^{2}$. 


\subsection{Diagonalization matrix}

After diagonalization of mass matrix the states for $a_{0}$ and $\pi$ mesons happen to be mixed. The eigenstates are defined as

$$
\begin{aligned}
a_{0} & =C_{a \tilde{a}} \tilde{a}+C_{a \tilde{\pi}} \tilde{\pi}, \quad \pi=C_{\pi \tilde{a}} \tilde{a}+C_{\pi \tilde{\pi}} \tilde{\pi}, \\
C_{a \tilde{a}} & =i C_{\pi \tilde{\pi}}=C_{+}, \quad C_{a \tilde{\pi}}=-i C_{\pi \tilde{a}}=-C_{-},
\end{aligned}
$$

with

$$
C_{ \pm}=\frac{1}{\sqrt{2}} \sqrt{1 \pm \frac{m_{a}^{2}-m_{\pi}^{2}}{\sqrt{\left(m_{a}^{2}-m_{\pi}^{2}\right)^{2}+\left(8 b^{\mu} k_{\mu}\right)^{2}}}} .
$$

We use the notation $\tilde{a}, \tilde{\pi}$, indicating that these states tend to $a_{0}, \pi$ when $b^{\mu}=0$.

One can see (Fig.2, left plot) that for $b_{\mu} b^{\mu}>0$ the growing chemical potential very quickly enforces the mixing between pions and isotriplet scalars so that distorted scalars happen to be involved into typical reactions of pion decay and pion formfactor. Yet with increasing of chemical potential the content of original pion states in distorted scalars and pseudoscalars diminishes.
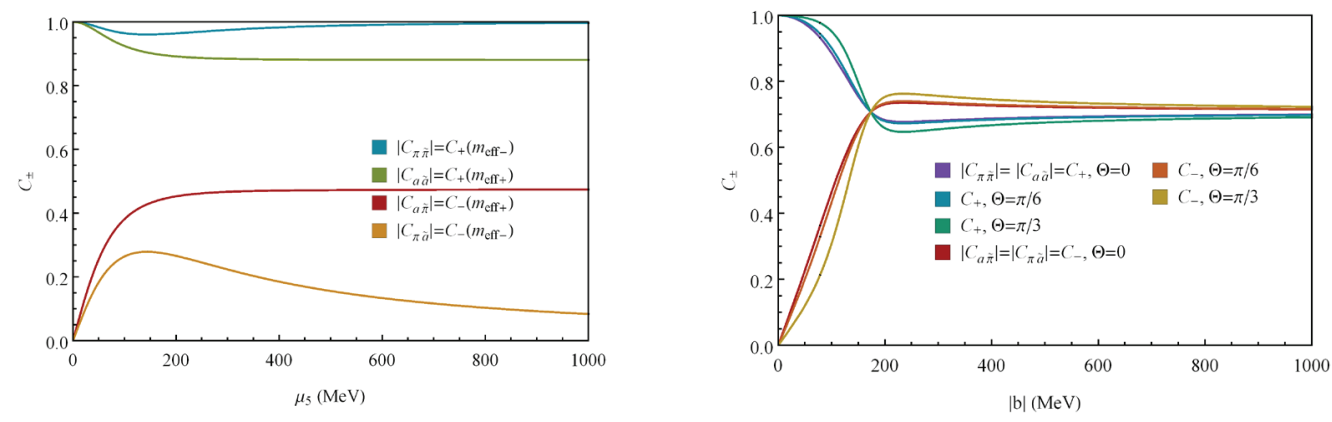

Figure 2. Left: mixing coefficients dependence on chemical potential $\mu_{5}$ for $|\vec{k}|=1000 \mathrm{MeV}$ in $b_{\mu} b^{\mu}>0$ region; right: mixing coefficients dependence on chemical potential $|b|$ for $|\vec{k}|=1000 \mathrm{MeV}$ in $b_{\mu} b^{\mu}<0$ region .

In turn, for $b_{\mu} b^{\mu}<0$ (Fig.2, right plot) the restoration of CS does not suppress the mixing of parity counterpartners and for increasing chemical potential tends to a nearly constant mixing coefficients with values in between 0 and 1 .

\subsection{Masses in CSB region with $b_{\mu} b^{\mu}>0$}

This is a region where CSB is enhancing and the $\tilde{a}_{0}$ and $\sigma$ mesons become more heavy with growing chemical potential.

Meantime the $\tilde{\pi}$-meson effective mass is slowly decreasing at rest and decreasing faster in flight with $|\vec{k}| \neq 0$. One can see how the $\tilde{\pi}$-meson reaches the massless point and further on its mass squared becomes negative, "tachyonic" which however does not cause any causality problems. One can check that the group velocity of these states remains less than the light velocity. 


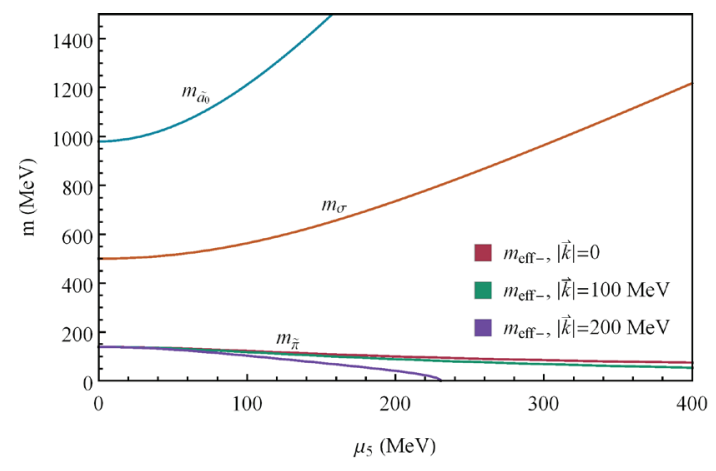

Figure 3. $\tilde{a}_{0}$-meson and $\tilde{\pi}$-meson effective mass dependence as well as $\sigma$-meson mass dependence on chemical potential $b=\mu_{5}$, for different values of $|\vec{k}|$ in $b_{\mu} b^{\mu}>0$ region.

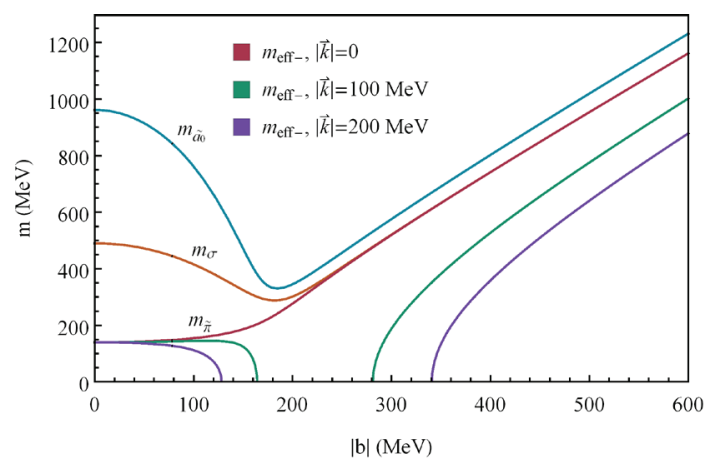

Figure 4. $\tilde{a}_{0}$-meson and $\sigma$-meson mass dependence on chemical potential $b, \tilde{\pi}$-meson effective mass dependence on $b$ for different values of $|\vec{k}|$ in $b_{\mu} b^{\mu}<0$ phase, the angle between $\vec{k}$ and $\vec{b}$ is $\theta=0$

\subsection{Masses in CSR region with $b_{\mu} b^{\mu}<0$}

In this region in the rest frame one can see clearly the CS restoration with merging masses of all scalars and pseudoscalars.

But in flight the behavior of pion masses is more peculiar. First pion masses vanish and then mass squared become negative. Next they reappear with positive mass squared and slowly approach to scalar masses in asymptotics. Thus the in-flight effect of chiral vector imbalance on pion spectrum deviates strongly from naive expectations.

5 The decays $\tilde{a}_{0}^{ \pm} \rightarrow \tilde{\pi}^{ \pm} \gamma, \tilde{\pi}^{0} \rightarrow \gamma \gamma, \tilde{a}_{0}^{0} \rightarrow \gamma \gamma$

After mixing $\pi, a_{0}^{0} \rightarrow \tilde{\pi}, \tilde{a}_{0}^{0}$ the decay $\tilde{a}_{0}^{ \pm} \rightarrow \tilde{\pi}^{ \pm} \gamma$ arises which breaks space parity and therefore is forbidden in vacuum.

with $q=|\vec{q}|$ being space momenta of scalars.

As well in the decay process $\pi^{0} \rightarrow \gamma \gamma$ an adjacent resonance decay $\tilde{a}_{0}^{0} \rightarrow \gamma \gamma$ emerges after mixing. From the plots for mixing coefficients we conclude that these processes are comparable in decay widths. 


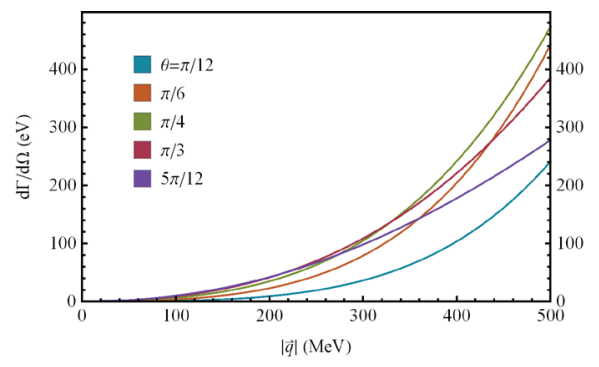

Figure 5. Decay width $a^{ \pm} \rightarrow \pi^{ \pm} \gamma, \mu_{5}=100 \mathrm{MeV}$
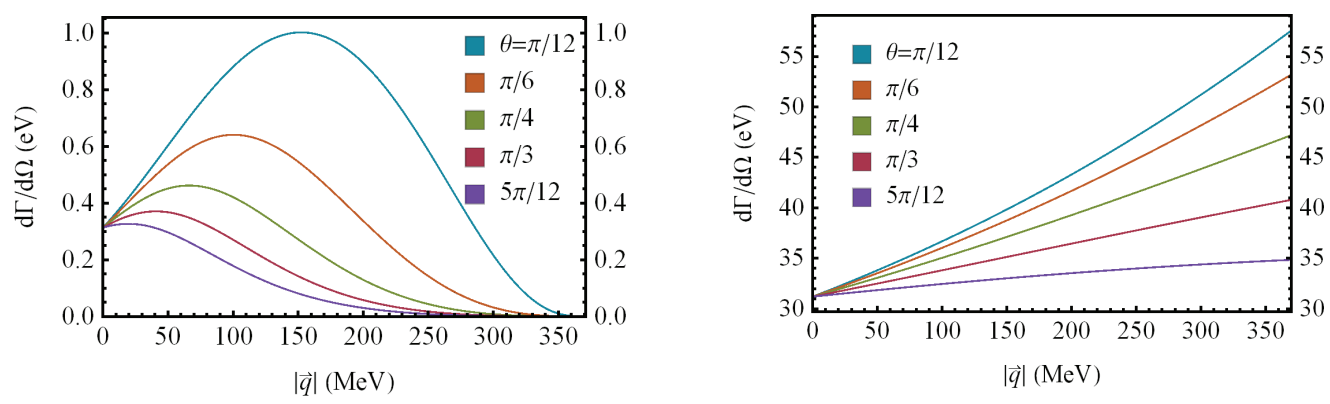

Figure 6. Decay widths; left: $\tilde{\pi}^{0} \rightarrow \gamma \gamma$, right: $\tilde{a}_{0}^{0} \rightarrow \gamma \gamma, \mu_{5}=100 \mathrm{MeV}$

For pseudoscalars and scalars in flight the speeds of decays are considerably increasing. The effects are opposite to the Lorentz retardation.

\section{Conclusions and outlook}

In this work we described a possibility of local parity breaking (LPB) emerging in a dense hot baryon matter (hadron fireball) in heavy-ion collisions at high energies. The phenomenology of LPB in a fireball is based on introducing a topological (axial) charge and a topological (chiral) chemical potential. Topological charge fluctuations transmit their influence to hadronic physics via an axial chemical potential. We suggested QCD-motivated sigma model for the description of isotriplet pseudoscalar and isoscalar and isotriplet scalar mesons in the body of a fireball. We conclude:

- Strong CP violation is quite a challenging possibility to be revealed in heavy-ion collisions both at high energy densities (temperatures) and being triggered by large baryon densities.

- However the existing theoretical arguments for arising CP violation in FINITE volumes are not well sufficient to calculate the production rate of $\mathrm{CP}$ violating nuclear processes.

- There are two ways to improve the discovery potential: firstly, to elaborate the recipes for experimentalists to detect peculiar effects generated in CP-odd background, secondly, to measure the production of the mass states without a firm CP parity. In both cases the chiral chemical potential method helps a lot in predictions.

- In addition we already suggested [14] the vector meson dominance model with chiral imbalance: the spectrum of massive vector mesons splits into three components with different polarizations and with different effective masses that can be used to detect local parity breaking. The proposed 
schemes for revealing local parity breaking helps to (partially) explain qualitatively and quantitatively the anomalous yield of dilepton pairs in the CERES, PHENIX, STAR, NA60, and ALICE experiments. Accordingly the identification of its physical origin might serve as a base for a deeper understanding of QCD properties in a medium under extreme conditions. Experimental collaborations should definitely check this possibility.

- Recently an interesting proposal was given in [21] to detect the LPB by measuring photon polarization asymmetry in the process $\pi^{ \pm} \gamma \rightarrow \pi^{ \pm} \gamma$. We extend this proposal indicating the resonance enhancement at energies comparable with the mass of $\tilde{a}_{0}^{ \pm}$scalars.

\section{Acknowledgements}

It is a pleasure to thank the organizers of the XXIII International Workshop on High Energy Physics and Quantum Field Theory, QFTHEP 2017 for a fruitful meeting and an excellent atmosphere. This work has been supported through grants FPA2013-46570, 2014-SGR-104 and Consolider CPAN. Funding was also partially provided by the Spanish MINECO under project MDM-2014-0369 of ICCUB (Unidad de Excelencia 'Maria de Maeztu'). A.A. and V.A. were supported by Grant RFBR projects 16-02-00348 and also got a financial support of SPbSU, in particular, by projects 11.41.417.2017, 11.41.415.2017, 11.42.697.2017 and 11.42.698.2017.

\section{References}

[1] P.Jacobs, D. Kharzeev, B. Muller, J. Nagle, K. Rajagopal and S. Vigdor, arXiv:0705.1930 [nuclex].

[2] J. -P. Blaizot, F. Gelis, J. -F. Liao, L. McLerran and R. Venugopalan, Nucl. Phys. A 873, 68 (2012) [arXiv:1107.5296 [hep-ph]].

[3] A. Andronic, D. Blaschke, P. Braun-Munzinger, J. Cleymans, K. Fukushima, L. D. McLerran, H. Oeschler and R. D. Pisarski, Nucl. Phys. A 837, 65 (2010) [arXiv:0911.4806 [hep-ph]].

[4] J. -P. Blaizot, Nucl. Phys. A 834, 515 C (2010) [arXiv:0911.5059 [hep-ph]].

[5] D. Kharzeev, R. D. Pisarski and M. H. G. Tytgat, Phys. Rev. Lett. 81, 512 (1998). D. E. Kharzeev, L. D. McLerran and H. J. Warringa, Nucl. Phys. A803, 227 (2008). K. Buckley, T. Fugleberg, A. Zhitnitsky, Phys. Rev. Lett., 84,4814 (2000).

[6] N. Ajitanand, S. Esumi, R. Lacey, P. Collaboration, et al., P-and CP-odd effects in hot and dense matter, in: Proc. of the RBRC Workshops, Vol. 96, 2010.

[7] P. Wurn et al. (CERES Collab.), Nucl. Phys. A, 5901-2, 103-116 (1995); CERES Collaboration (Agakichiev, G. et al.) Phys. Rev. Lett. 75 , 1272 (1995); Phys. Lett. B 422, 405(1998); Eur. Phys. J. C 41, 475(2005).

[8] R. Arnaldi et al. (NA60 Collab.), Phys. Rev. Lett., 96 , 162302 (2006).

[9] A. Adare et al. (PHENIX Collab.), Phys. Rev. C, 81, 034911 (2010).

[10] G. Agakichiev et al. (HADES Collab.), Phys. Rev. Lett., 98, 052302 (2007). Phys. Lett. B, 663, 43-48 (2008).

[11] K. O. Lapidus, V. M. Emel'yanov, Phys. Part. Nucl., 40, 29 (2009).

[12] I. Tserruya, Electromagnetic Probes, arXiv: 0903.0415; G. E. Brown, M. Rho, Phys. Rev. Lett., 66,2720-2723 (1991).

[13] A. A. Andrianov, V. A. Andrianov, D. Espriu, X. Planells, Theor.Math.Phys., 170, 17 (2012); A. A. Andrianov, V. A. Andrianov, Theor.Math.Phys., 185, 1370 (2015). 
[14] A. A. Andrianov, V. A. Andrianov, D. Espriu and X. Planells, Phys. Lett. B 710230 (2012); Proc.Sci., QFTHEP, 025 (2013); Phys. Rev. D, 90,034024 (2014).

[15] D. Kharzeev, Y. Kikuchi and R. Meyer, arXiv:1610.08986 [cond-mat.mes-hall].

[16] A. Sorin and O. Teryaev, Nucl. Part. Phys. Proc. 273-275, 2587 (2016). doi:10.1016/j.nuclphysbps.2015.09.468 .

[17] K. Landsteiner, Acta Phys. Polon. B 47, 2617 (2016) doi:10.5506/APhysPolB.47.2617 [arXiv:1610.04413 [hep-th]].

[18] A. A. Andrianov, D. Espriu and X. Planells, Eur. Phys. J. C 73:2294 (2013).

[19] J. Wess and B. Zumino, Phys. Lett. 37B, 95 (1971); E. Witten, Nucl. Phys. B223, 422 (1983); A. A. Andrianov, V. A. Andrianov, V. Yu. Novozhilov, and Yu. V. Novozhilov, Theor. Math. Phys.,70, 43 (1987).

[20] V. V. Braguta et al., Phys. Rev. D 93, 034509 (2016); V. V. Braguta et al. AIP Conf. Proc. 1701, 060002 (2016); V. V. Braguta, A. Yu. Kotov, Phys. Rev. D93, 105025 (2016).

[21] Kawaguchi M., Harada M., Matsuzaki S., Ouyang R.M., Phys. Rev. C 95, 065204 (2017) . 